\title{
Aprovechamiento de los subproductos del mango, como fuente de compuestos bioactivos, para la elaboración de rollos comestibles
}

\section{Use of the mango by-products, as source of bioactive compounds, for the preparation of edible rolls}

\author{
Karla Daniela Rubiano-Charry ${ }^{1 *}$; Héctor José Ciro-Velásquez ${ }^{2}$; Iván Darío Aristizabal-Torres ${ }^{3}$
}

IIng. Química, M.Sc., Joven investigador. Universidad Nacional de Colombia - Colciencias. Medellín - Antioquia, Colombia; e-mail: kdrubianoc@unal.edu.co; (Dhttps://orcid.org/0000-0002-2357-570X

Ing. Mecánico, M.Sc., Ph.D. Universidad Nacional de Colombia, Sede Medellín, Departamento de Ingeniería Agrícola y Alimentos. Medellín - Antioquia, Colombia; e-mail: hjciro@unal.edu.co; (Dhttps://orcid.org/0000-0002-4398-0470

${ }^{3}$ Ing. Agrícola, D.Sc. Universidad Nacional de Colombia, Sede Medellín, Departamento de Ingeniería Agrícola y Alimentos. Medellín - Antioquia, Colombia; e-mail: idaristi@unal.edu.co; Dhttps://orcid.org/0000-0003-1913-6832

*autor para correspondencia: kdrubianoc@unal.edu.co

Cómo citar: Rubiano-Charry, K.D.; Ciro-Velásquez, H.J.; Aristizabal-Torres, I.D. 2019. Aprovechamiento de los subproductos del mango, como fuente de compuestos bioactivos, para la elaboración de rollos comestibles. Rev. U.D.C.A Act. \& Div. Cient. 22(2):e1078. http://doi.org/10.31910/rudca.v22.n2.2019.1078

Artículo de acceso abierto publicado por Revista U.D.C.A Actualidad \& Divulgación Científica, bajo una licencia Creative Commons CC BY-NC 4.0

Recibido: Noviembre 14 de 2018

Aceptado: Noviembre 15 de 2019

Editado por: Rita María Ávila G. de Hernández

\section{RESUMEN}

El mango es una fruta con alto valor nutricional, posee gran cantidad de vitaminas, fibra, antioxidantes y otros micronutrientes. La cáscara y la semilla del mango son consideradas residuos, pero estudios demuestran que son fuente de compuestos bioactivos. Por lo anterior, el objetivo de esta investigación fue desarrollar un producto alimentario a base de pulpa de mango Mangifera indica L., con la adición de componentes bioactivos (polifenoles y vitamina $\mathrm{C}$ ), presentes en la cáscara. Se evaluó la extracción por fluidos supercríticos de polifenoles, posterior al secado al vacío $\left(40^{\circ} \mathrm{C}, 33 \mathrm{mbar}\right.$, durante 7,5 horas) y molienda criogénica de la cáscara de mango. Los resultados indicaron un rendimiento del proceso de extracción de 56,67\%, por lo que se estableció adicionar directamente la cáscara de mango deshidratada y molida al producto alimentario. Posteriormente, se determinó la formulación para elaborar un rollo comestible a partir de pulpa de mango, mediante un diseño factorial, variando la adición de ácido ascórbico (0,3-0,5\% p/ p) y cáscara de mango deshidratada $(1-3 \% \mathrm{p} / \mathrm{p})$, teniendo como variables de respuesta propiedades fisicoquímicas y sensoriales. Se estableció como formulación adecuada: 0,5\% ácido ascórbico y $1 \%$ cáscara de mango, así como la adición de ácido cítrico 0,5\%, azúcar 3,5\%, maltodextrina 10\%, CMC 1\% y pulpa 83,5\%; los rollos fueron obtenidos por secado convectivo hasta humedad de $24 \%$ b.h. Lo anterior permitió obtener un producto con calidad apropiada ( $\mathrm{pH} 4,39$, aw 0,8275, humedad 23,47\% b.h, parámetros de color L* 46,9, a* 2,86, b* 37,89 y calidad sensorial alta), además de contener sustancias bioactivas: vitamina C $(48,38 \mathrm{mg} / 100 \mathrm{~g})$ y polifenoles (capacidad antioxidante 63,63\%).

Palabras clave: fruta; Mangifera indica; producto alimenticio; diseño; subproducto (Thesaurus UNESCO). 


\section{ABSTRACT}

Mango is a fruit with high nutritional value, it has a large amount of vitamins, fiber, antioxidants and other micronutrients. The mango peel and seed are considered waste, but studies show that they are a source of bioactive compounds. Therefore, the objective of this study was to develop a food product (edible rolls) based on mango pulp (Mangifera indica L.) with the addition of bioactive components (polyphenols and Vitamin C) present in the mango peel. The extraction of polyphenols was done by supercritical fluids, after vacuum drying $\left(40^{\circ} \mathrm{C}, 33 \mathrm{mbar}\right.$, for 7.5 hours $)$ and cryogenic grinding of the mango peel. The yield of the extraction process was $56.67 \%$, so it was established to add the dehydrated and ground mango husk directly to the food product. Subsequently, the formulation to produce an edible roll from mango pulp was determined, by means of a factorial design, varying the addition of ascorbic acid $(0.3-0.5 \% \mathrm{w} / \mathrm{w})$ and dehydrated mango peel $(1-3 \% \mathrm{w} / \mathrm{w})$, having as response variables physicochemical and sensory properties. It was established as a suitable formulation: $0.5 \%$ ascorbic acid and $1 \%$ mango peel, as well as the addition of citric acid $0.5 \%$, sugar $3.5 \%$, maltodextrin $10 \%$, CMC $1 \%$ and pulp $83.5 \%$; the rolls were obtained by convective drying until to reach a moisture content less than $24 \%$ w.b. This allowed obtaining a product with appropriate quality ( $\mathrm{pH} 4.39$, aw 0.8275 , moisture content $23.47 \%$ w.b, color parameters $\mathrm{L} * 46.9, \mathrm{a} * 2.86, \mathrm{~b} * 37.89$ and high sensory quality), besides to containing bioactive substances: vitamin C $(48.38 \mathrm{mg} /$ $100 \mathrm{~g}$ ) and polyphenols (antioxidant capacity 63.63\%).

Keywords: fruit; Mangifera indica; food product; design; by-product.

\section{INTRODUCCIÓN}

A nivel mundial, las frutas comprenden una cadena de gran importancia en la agroindustria y en el desarrollo de diversos productos alimentarios, debido a sus propiedades nutricionales, al ser fuente de vitaminas, minerales, ácidos grasos, antioxidantes, entre otros, los cuales, son fundamentales para la salud humana (Oliveira et al. 2018).

En Colombia, la cadena hortofrutícola carece de sistemas tecnológicos, que permitan la diversificación en el desarrollo de productos y aprovechamiento de subproductos, afectando la productividad y el crecimiento económico (Orjuela et al. 2017).

Actualmente, la producción primaria e industrialización de frutas genera subproductos en grandes cantidades, que causan contaminación que originan problemas ambientales, además de las pérdidas económicas, al no ser utilizados eficientemente. Durante el procesamiento del mango, por ejemplo, en la elaboración de jugos, se emplean solo pequeñas porciones del mismo y cantidades considerables de cáscaras y semillas son descartadas como desechos industriales (García et al. 2015), los cuales, se estiman alrededor de $75000 \mathrm{~m}$ T, en todo el mundo (Dorta et al. 2011). Se ha encontrado que la cáscara constituye un $15-20 \%$ y la semilla entre $35-60 \%$ del peso total del mango (Jahurul et al. 2015).
Cabe resaltar que diferentes estudios han encontrado que los subproductos del mango, como la cáscara y la semilla, son fuente importante de compuestos bioactivos, entre los que se incluyen carotenoides, flavonoides, antocianinas, polifenoles, pectina, manguiferina, fibra dietaria y ácidos grasos poliinsaturados (García et al. 2015). También, se reporta que los componentes bioactivos presentes poseen alta actividad antioxidante, tanto en cáscara como en semilla, inclusive, mayor que en la pulpa (Sumaya et al. 2012). Así, estos componentes pueden llegar a ser ingredientes de alto valor nutricional/funcional y de importancia para la industria alimentaria, cosmética o farmacéutica. La extracción por Soxhlet, con solventes es el método más empleado para la extracción de componentes bioactivos; sin embargo, este es un proceso lento que tiene repercusiones negativas al ambiente; otra alternativa, es el uso de fluidos supercríticos, que corresponde a una tecnología verde y genera mayores rendimientos (Wen et al. 2018).

Por su parte, en Colombia, se siembran diversas variedades de mango, siendo que el 39\% del área cultivada corresponde a plantaciones silvestres de variedad hilacha, Tommy Atkins (20\%), Keitt (11\%) y mango de azúcar (5\%) (Corrales et al. 2014).

A nivel industrial, el mango ha sido transformado en una gran variedad de productos, como jugos, polvos, purés, trozos mínimamente procesados y rodajas deshidratadas (Dak et al. 2007; Djantou et al. 2011; Sriwimon \& Boonsupthip, 2011; Ledeker et al. 2014; Sogi et al. 2015; Teixeira et al. 2013); sin embargo, estos alimentos solo aprovechan la pulpa, sin generar valor agregado a los subproductos, como la cáscara y la semilla.

De acuerdo con lo anterior, el objetivo de esta investigación fue desarrollar un producto comestible a base de pulpa de mango (Mangifera indica L.), variedad azúcar, con la adición de componentes bioactivos, provenientes de su cáscara. Cabe resaltar que el producto enrollable es una lámina a partir de la pulpa de mango, con propiedades texturales, que permiten enrollarse sobre sí misma. Adicionalmente, a pesar de que el mango es una fruta de gran consumo en el mundo, según las referencias bibliográficas, no se tiene información disponible para productos similares y con el aprovechamiento de su cáscara.

\section{MATERIALES Y MÉTODOS}

Materias primas y localización. Se empleó mango de azúcar (Mangifera indica), procedente de un cultivo comercial, en estado de maduración intermedia (grado 4), cumpliendo con lo establecido por el Instituto Colombiano de Normas Técnicas (2003). El proceso de deshidratación, la extracción por fluidos supercríticos y la caracterización de las propiedades fisicoquímicas de la materia prima y el producto final, se llevó a cabo en el Laboratorio de Procesos Agrícolas de la Universidad Nacional de Colombia, sede Medellín.

Caracterización de la materia prima (mango). Se evaluaron las siguientes características fisicoquímicas y bromatológicas de la pulpa de mango: 
Actividad de agua (aw). Evaluada mediante un higrómetro de punto de rocío, a $25^{\circ} \mathrm{C}$ (serie Aqualab 3TE, Decagon, Devices, Pullman, WA, USA).

Color. Se empleó la metodología sistema de espacio de color uniforme CIE L*a*b* y h (ángulo de tono). La medición, se realizó con un espectrofotómetro de esfera X- Rite con iluminante D65 y $10^{\circ}$ de ángulo de observación.

Contenido de humedad. Se determinó de acuerdo con la metodología AOAC 925.45/97.

Sólidos solubles totales ( ${ }^{\circ}$ Brix). Se utilizó un refractómetro digital (Hanna Instruments 96801), que emplea el fenómeno de la refracción de la luz, para determinar el porcentaje de solidos solubles totales e índice de refracción.

pH. Se determinó de acuerdo con lo establecido por el Instituto Colombiano de Normas Técnicas (1999), empleando un potenciómetro por inmersión de electrodo en la muestra (titulador automático Hanna pH 211).

Cenizas. Se empleó el método gravimétrico. Se pesó un gramo de muestra y se incineró en mufla a $500^{\circ} \mathrm{C}$ por 5 horas.

Proteína. Se determinó de acuerdo con el método Microkjeldhal AOAC 954.01.

Grasa total. Se determinó mediante técnica de extracción - AOAC 920.39 .

Viscosidad. Se midió usando un reómetro BrookField DV-III Ultra a temperatura de $25^{\circ} \mathrm{C}$, considerando una máxima velocidad de corte a 200rpm. El valor de la viscosidad fue determinado mediante un análisis de los reogramas o curvas del flujo del producto (Frascareli et al. 2012).

Extracción e identificación de compuestos bioactivos presentes en la cáscara de mango. Para esta actividad, se consideró la técnica de fluidos supercríticos previo a un proceso de adecuación de la materia prima, mediante secado al vacío y molienda criogénica.

Secado al vacío. Para la deshidratación de la cáscara de mango, se empleó una estufa a vacío Memmert (VO29), fijando la temperatura en $40^{\circ} \mathrm{C}$, presión de $33 \mathrm{mbar}$, variando el tiempo de secado en 5,5 y 7,5horas, tomando como variable de respuesta el contenido de humedad final del producto.

Molienda criogénica. Una vez terminada la etapa de secado, la muestra se dejó reposar hasta alcanzar la temperatura ambiente; seguidamente, se ultracongeló hasta $-40^{\circ} \mathrm{C}$ y se procedió a moler, empleando un molino de cuchillas. El producto en polvo fue pasado por la malla 200 y su retenido fue usado en las diferentes extracciones.

Extracción por fluidos supercríticos. La extracción, se llevó a cabo en el sistema de extracción supercrítico (Waters ${ }^{\circledR}$ MV-10). Para ello, se colocaron tres gramos de muestra de cáscara de mango pretratada (deshidratada y molida), dentro de la celda de extracción. Se empleó un cosolvente polar $(20 \%$ etanol) para aumentar la recuperación de los componentes bioactivos y las condiciones de operación se establecieron, de acuerdo con evaluaciones preliminares, en 300 bares y $40^{\circ} \mathrm{C}$, con $13 \mathrm{~mL} / \mathrm{min}$ flujo de $\mathrm{CO}_{2}$, extracción estática de 15 minutos y extracción dinámica de 75 minutos (García et al. 2015). Los compuestos bioactivos extraídos, polifenoles y vitamina C, se cuantificaron en $\mathrm{mg} / 100 \mathrm{~g}$ de producto.

Desarrollo de producto a partir de pulpa de mango y adición de compuestos bioactivos. Se realizaron ensayos preliminares para definir la formulación base para la elaboración de rollos comestibles, a partir de pulpa de mango, así como las condiciones del proceso. Para ello, se evaluaron agentes texturizantes (maltodextrina y CMC), para lograr las propiedades texturales deseadas (producto tipo enrollable) y la adición de agentes antioxidantes (ácido cítrico), para evitar el pardeamiento del producto.

Posteriormente, se evaluó la adición de los compuestos bioactivos presentes en la cáscara de mango (previamente obtenida) en el producto alimentario, mediante un diseño factorial $2^{2}$, con tres repeticiones, teniendo como factores el porcentaje de adición de ácido cítrico $(0,3-0,5 \% \mathrm{p} / \mathrm{p})$ y de cáscara de mango deshidratada $(1-3 \% \mathrm{p} / \mathrm{p})$. Se establecieron, como variables de respuesta para la mezcla $\mathrm{pH}$, color, acidez y viscosidad y para el producto final humedad, sólidos solubles, expresados como ${ }^{\circ}$ Brix, actividad de agua (aw), color y textura. Las diferentes formulaciones fueron deshidratadas mediante secado convectivo, a $50^{\circ} \mathrm{C}$, durante 4 horas, condiciones definidas mediante evaluaciones preliminares.

Análisis de textura. Se realizó una prueba de tensión a punto de fallo en las muestras, usando un analizador da textura (TA- XT2i, Estable Micro Systems, Godalming, Reino Unido), equipado con una celda de carga de 50kg. Para el análisis, los rodillos tensores se fijaron en la base del analizador de textura y en la celda de carga. La distancia de deformación de la muestra se estableció en $50 \mathrm{~mm}$ y la velocidad de la prueba fue de $2 \mathrm{~mm} / \mathrm{s}$. La fuerza de ruptura, se tomó como la máxima fuerza $(\mathrm{N})$, requerida para romper la muestra (Romero et al. 2014). Las dimensiones de las muestras a analizar fueron en promedio, espesor de $2 \mathrm{~mm}$ y ancho de $1,5 \mathrm{~cm}$.

Caracterización del producto final. Se determinaron las siguientes características fisicoquímicas y tecnofuncionales: $\mathrm{pH}$, ${ }^{\circ} \mathrm{Brix}$, aw, \%humedad, acidez, color, análisis textural, contenido de vitamina C, capacidad antioxidante (determinación de DPPH), temperatura de transición vítrea y análisis sensorial.

Vitamina C. El análisis, se realizó utilizando un sistema de HPLC. Los resultados, se expresaron como miligramos de vitamina $C$ por 100g del producto (Guiamba et al. 2016).

Capacidad antioxidante (determinación de DPPH). Para determinar esta propiedad, se empleó la ecuación 1, en el que cada absorbancia fue medida a una longitud de onda de $51 \mathrm{~nm}$, en un espectrofotómetro (Londoño, 2012). 
\%Inhibición $=\frac{\mathrm{Abs}_{\text {referencia }}-\mathrm{Abs}_{\text {muestra }}}{\mathrm{Abs}_{\text {referencia }}} * 100$

ecuación 1

Temperatura de transición vítrea. Se determinó mediante un calorímetro diferencial de barrido Q2000 (TA Instruments), para lo cual, se tomaron $10 \mathrm{mg}$ de muestra (rollo de mango), en un crisol de aluminio herméticamente sellado. La velocidad de calentamiento y enfriamiento modulado se fijó a $2^{\circ} \mathrm{C} /$ min en un rango de temperatura de $-70^{\circ} \mathrm{C}$ a $250^{\circ} \mathrm{C}$ y periodo de oscilación de $60 \mathrm{~s}$.

Análisis sensorial. Se realizó un análisis sensorial descriptivo cuantitativo, mediante siete jueces entrenados, de acuerdo con las NTC 3932 y 5328. Las características sensoriales evaluadas fueron olor, sabor, apariencia y calidad general. Los descriptores fueron calificados en una escala de intensidad de 10 puntos, donde 0 es ausencia, valores entre 0,1 - 2,9 muy leve; 3 - 4,9 leve; 5 - 6,9 moderada; 7 - 8,9 marcada; 9 - 10 muy marcada.

Análisis estadístico. Cada tratamiento y sus mediciones, se realizaron por triplicado. Los datos fueron analizados mediante un análisis de varianza multivariado (ANAVA) y se aplicaron pruebas de diferencias mínimas significativas (LSD) de Fisher, para comparar las diferencias entre las medias, con un nivel de confianza del 95\%; lo anterior, empleando el software STATGRAFIT CENTURION® (versión XVI).

\section{RESULTADOS Y DISCUSIÓN}

Caracterización de la materia prima (mango). Los resultados de la caracterización fisicoquímica y bromatológica del mango variedad azúcar en estado de maduración 4 , se presentan en la tabla 1. En el análisis bromatológico de la pulpa de mango, se obtuvieron resultados similares a lo reportado por Corrales et al. (2014), aunque difiere en el contenido de cenizas, posiblemente, por el grado de maduración evaluado. Cabe resaltar, que se eligió este estado de madurez intermedia, teniendo en cuenta el producto alimentario a desarrollar (enrollable comestible) y la necesidad de incorporar una nota dulce, a nivel sensorial y característica al mango.

Extracción de compuestos bioactivos presentes en la cáscara de mango. Durante el proceso de secado al vacío de las cáscaras de mango, a $40^{\circ} \mathrm{C}$ y 33 mbar, el producto presentó humedades de 9,21 y 4,21\% b.h., para un tiempo de secado de 5,5 y 7,5 horas, respectivamente. Teniendo en cuenta los requerimientos de humedad de la muestra para la técnica de extracción por fluidos supercríticos, usando el equipo tecnológico de referencia, se estableció emplear el pretratamiento de secado al vacío durante 7,5 horas. Posteriormente, la extracción, mediante fluidos supercríticos presentó un rendimiento de $56,67 \%$, por lo que se convierte en una alternativa poco viable para escalar, a nivel industrial, considerando también que el tiempo del proceso fue prolongado (90 min). Debido a lo anterior, se estableció adicionar los compuestos bioactivos en el producto alimentario, a partir de la cáscara de mango deshidratada y molida de forma criogénica, es decir, se adicionó el producto de la molienda de la cáscara retenido en la malla 200 (partículas de tamaño mayor a $74 \mu \mathrm{m})$.

Desarrollo de producto a partir de pulpa de mango y adición de compuestos bioactivos. En la tabla 2, se observa la formulación base seleccionada para el producto a desarrollar, de acuerdo con lo obtenido en los ensayos preliminares, en la cual, se incluyen agentes texturizantes y antioxidantes, que permiten obtener las características sensoriales deseadas.

Por su parte, la tabla 3 muestra el promedio de los resultados obtenidos en la caracterización de las propiedades fisicoquímicas, para las diferentes formulaciones evaluadas en el desarrollo del producto. La actividad de agua estuvo en un rango entre 0,73 - 0,87, valores característicos para productos de humedad intermedia, pero cabe resaltar que está por encima de la humedad obtenida en la

Tabla 1. Caracterización fisicoquímica y bromatológica de la pulpa de mango variedad azúcar en estado de maduración 4.

\begin{tabular}{|l|l|}
\hline \multicolumn{1}{|c|}{ Parámetro } & \multicolumn{1}{c|}{ Valor* } \\
\hline Actividad de agua & $0,98 \pm 0,0005$ \\
\hline \multirow{4}{*}{ Color (pulpa) } & $\mathrm{L}^{*}=42,28 \pm 0,26$ \\
\cline { 2 - 2 } & $\mathrm{a}^{*}=3,56 \pm 0,04$ \\
\cline { 2 - 2 } & $\mathrm{b}^{*}=18,46 \pm 0,29$ \\
\cline { 2 - 2 } & $\mathrm{h}^{\circ}=79,07 \pm 0,07$ \\
\hline Humedad (\%) & $88,54 \pm 1,38$ \\
\hline Sólidos Solubles Totales ( ${ }^{\circ}$ Brix $)$ & $10,13 \pm 0,06$ \\
\hline \multirow{2}{*}{$\mathrm{H}$} & $3,71 \pm 0,01$ \\
\hline Cenizas $(\%)$ & $2,96 \pm 0,00$ \\
\hline Proteína $(\%)$ & $0,0 \pm 0,00$ \\
\hline Grasa total $(\%)$ & $0,0 \pm 0,00$ \\
\hline Viscosidad $(\mathrm{Cp})$ & $438,00 \pm 11,14$ \\
\hline Vitamina C $(\mathrm{mg} / 100 \mathrm{~g})$ & $32,45 \pm 1,05$ \\
\hline
\end{tabular}

Los resultados se expresan con media y desviación estándar $(\mathrm{n}=3)$. 
Tabla 2. Formulación de los rollos de mango desarrollados.

\begin{tabular}{|c|c|}
\hline Componente de la formulación & $\%$ \\
\hline Ácido ascórbico & $0,3-0,5$ \\
\hline Cáscara de mango & $1-3$ \\
\hline Ácido cítrico & 0,5 \\
\hline Azúcar & 3,5 \\
\hline Maltodextrina & 10 \\
\hline CMC & 1 \\
\hline
\end{tabular}

deshidratación osmótica del mango (Guiamba et al. 2016). Los sólidos solubles totales, expresados como grados ${ }^{\circ}$ Brix, presentaron valores entre 63,20 - 75,13, resultados mayores que la pulpa fresca, debido, principalmente, por la adición de carbohidratos, como maltodextrina, CMC y azúcar. La luminosidad L* del producto varió entre 24,62 -
40,40, mostrando que, para algunas formulaciones, esta propiedad fue similar a la pulpa fresca $(42,28)$, a pesar del tratamiento térmico efectuado. Con respecto a los parámetros de color $\mathrm{a}^{*} \mathrm{y} \mathrm{b}^{*}$, presentaron valores entre 4,54 - 6,70 y 16,51 - 25,61, respectivamente, los cuales, fueron mayores al comparar con la fruta fresca.

Tabla 3. Caracterización de los enrollables de mango desarrollados bajo diferentes formulaciones.

\begin{tabular}{|c|c|c|c|c|}
\hline $\begin{array}{c}\text { Cáscara mango } \\
\text { deshidratada (\%) }\end{array}$ & $\mathbf{3}$ & $\mathbf{1}$ & $\mathbf{1}$ & $\mathbf{3}$ \\
\hline Ácido ascórbico (\%) & 0,5 & 0,5 & 0,3 & 0,3 \\
\hline Acidez (\%) & $25,83 \pm 0,15$ & $25,23 \pm 0,30$ & $16,43 \pm 0,40$ & $15,47 \pm 1,06$ \\
\hline pH & $3,75 \pm 0,13$ & $4,54 \pm 0,27$ & $5,51 \pm 0,19$ & $4,49 \pm 0,27$ \\
\hline Humedad (\%, b.h) & $22,79 \pm 0,36$ & $22,82 \pm 0,43$ & $20,82 \pm 0,52$ & $21,65 \pm 0,57$ \\
\hline aw & $0,8752 \pm 0,0030$ & $0,8437 \pm 0,0024$ & $0,7520 \pm 0,0078$ & $0,7308 \pm 0,0039$ \\
\hline SST ('Brix) & $71,80 \pm 1,37$ & $63,20 \pm 0,17$ & $63,47 \pm 1.09$ & $75,13 \pm 0,57$ \\
\hline Fuerza de Falla (N) & $28,40 \pm 1,95$ & $22,25 \pm 1,92$ & $23,12 \pm 1,21$ & $13,32 \pm 0,95$ \\
\hline Viscosidad (mPa.s) & $13,48 \pm 0,73$ & $6,91 \pm 0,98$ & $7,90 \pm 0,87$ & $13,12 \pm 0,74$ \\
\hline L* & $24,62 \pm 0,30$ & $40,40 \pm 0,15 * *$ & $26,91 \pm 0,21$ & $34,81 \pm 0,42$ \\
\hline $\mathbf{a}^{*}$ & $5,85 \pm 0,01$ & $6,70 \pm 0,03$ & $4,96 \pm 0,01$ & $4,54 \pm 0,02$ \\
\hline $\mathbf{b}^{*}$ & $20,70 \pm 0,18$ & $25,61 \pm 0,32$ & $16,51 \pm 0,10$ & $23,35 \pm 0,25$ \\
\hline
\end{tabular}

Los resultados se expresan con media y desviación estándar $(n=3)$. Promedios denotados con $* *$ presentaron diferencias significativas $(\mathrm{p}<0,05)$ según prueba LSD de Fisher.

Similarmente, los resultados para el análisis de textura, mostraron que la fuerza de fallo varió entre 13,32 - 28,40N, lo que evidencia que el producto desarrollado no requiere una alta aplicación de fuerza para su ruptura. Por su parte, el contenido de humedad estuvo entre 20,82 - 22,82\%, valores característicos para el tipo de producto y en relación con la mezcla, la viscosidad varió entre $6,91-13,48 \mathrm{mPa}$. (comportamiento newtoniano); la acidez varió entre 15,47 - 25,83\% y el $\mathrm{pH}$, entre 3,75 - 5,51, que fueron mayores que en la pulpa fresca.

Los resultados del análisis estadístico solo mostraron diferencias significativas $(p<0,05)$ para la variable $L^{*}$, pues las demás presentaron un valor $\mathrm{p}>0,05$. De acuerdo con lo anterior, se eligió, como la formulación adecuada, la que permitía obtener un menor pardeamiento en el producto final, es decir, aquella con mayor valor en el parámetro L*. Por ello, la formulación seleccionada para el desarrollo del producto consistió en: 1\% de cáscara de mango deshidratada y molida, junto con $0,5 \%$ de ácido ascórbico, así como la adición de ácido cítrico 0,5\%, azúcar 3,5\%, maltodextrina 10\%, CMC $1 \%$ y pulpa de mango $83,5 \%$.

Caracterización del producto final. En la tabla 4. se muestra la caracterización fisicoquímica del producto desarrollado bajo la formulación seleccionada. El contenido de humedad y la actividad de agua $\left(a_{w}\right)$ evidencian que es un producto de humedad intermedia, que tienen la ventaja de permitir ser almacenados a temperatura ambiente; su vida útil es mayor con respecto al alimento en fresco y contienen sólidos disueltos, que inhiben el crecimiento de bacterias, mohos, levaduras y controlan la actividad enzimática indeseable (Prabhakar, 2014). Estas características de humedad intermedia en el producto terminado evidencian la plasticidad del producto desarrollado, propiedad reológica y textural destacada para la descripción del enrollable; lo anterior, también se evidencia en el análisis de textura. 
Tabla 4. Propiedades fisicoquímicas y funcionales del producto desarrollado.

\begin{tabular}{|c|c|c|}
\hline Parámetro & Mezcla & Producto final \\
\hline $\mathrm{pH}$ & $3,70 \pm 0,08$ & $4,39 \pm 0,15$ \\
\hline${ }^{\circ}$ Brix & $23,40 \pm 1,05$ & $63,30 \pm 1.39$ \\
\hline aw & $0.99 \pm 0,00$ & $0,82 \pm 0,00$ \\
\hline \%Humedad (b.h) & $73,76 \pm 1,23$ & $23,47 \pm 0,98$ \\
\hline \%Acidez & $26,83 \pm 0,57$ & - \\
\hline L & - & $46,90 \pm 0,05$ \\
\hline a & - & $2,86 \pm 0,02$ \\
\hline b & - & $37,89 \pm 0,11$ \\
\hline Vextura: Fuerza de falla (N) & - & $22,77 \pm 2,76$ \\
\hline Vitamina C (mg/100g) & - & $48,38 \pm 0,93$ \\
\hline Capacidad antioxidante (\%) & - & $63,62 \% \pm 4,70 \%$ \\
\hline
\end{tabular}

Los resultados se expresan con media y desviación estándar $(\mathrm{n}=3)$.

El enrollable de mango presentó parámetros de color similares a los valores de la pulpa fresca, como se observa en las tablas 1 y 4 , donde la principal variación fue en el parámetro b*, puesto que el producto desarrollado tomó una tonalidad de amarillo más intenso. En la tabla 4, también se observa el contenido de vitamina $C$ en el producto y la capacidad antioxidante de este, medida mediante metodología DPPH. Con respecto al contenido de vitamina $\mathrm{C}$ fue de, aproximadamente, $28 \%$, mayor con respecto al fruto fresco $(48,38 \mathrm{mg} / 100 \mathrm{~g}$ frente a $32,45 \mathrm{mg} / 100 \mathrm{~g}$ para el producto y la pulpa, respectivamente). De igual forma, este resultado fue mayor a lo reportado por Mendoza-Corviz et al. (2017), para un producto en polvo, elaborado a base de pulpa de mango var. Hilacha y lactosuero, así como lo encontrado por Guiamba et al. (2015), para mango var. Tommy Atkins deshidratado; estos resultados muestran que la adición de cáscara de mango deshidrata contribuyó al aumento del contenido de este bioactivo, a pesar del tratamiento térmico aplicado. Por su parte, la capacidad antioxidante en el producto desarrollado es similar a lo reportado por Ajila et al. (2010), quienes también evaluaron el polvo de cáscara de mango, aplicada para el desarrollo de macarrones.

En la figura 1, se observa los resultados de la caracterización sensorial del producto. De acuerdo con el Análisis Descriptivo Cuantitativo, los enrollables de mango presentan una apariencia brillante marcada y leve intensidad de presencia de partículas en su superficie, asociado a la cáscara de mango. El olor y sabor frutal, sabor a cáscara de mango y textura cohesiva, presentaron una intensidad marcada. Los resultados para los diferentes factores evaluados permitieron que la calidad del producto fuera alta, con un puntaje de 9,1. En consecuencia, el producto desarrollado presenta un perfil sensorial adecuado.

La figura 2 muestra el termograma del producto, obtenido mediante análisis por calorimetría diferencial de barrido (DSC). De acuerdo con los resultados, la temperatura de transición vítrea $(\mathrm{Tg})$, para el rollo de mango, es aproximadamente $68,58^{\circ} \mathrm{C}$, comenzando en $68,25^{\circ} \mathrm{C}$ y con un punto final en $73,40^{\circ} \mathrm{C}$. El alto valor en la $\mathrm{Tg}$ es debido a la adición de maltodextrina y $\mathrm{CMC}$ en la formulación, que tienen temperaturas de transición vítrea de $162^{\circ} \mathrm{C}$ y $78,21^{\circ} \mathrm{C}$, respectivamente (Kurozawa et al. 2009; Akhtar et al. 2018); además, al ser compuestos de alto peso molecular, permiten aumentar la Tg (Rahman, 2009). De acuerdo con los resultados, el producto se puede almacenar a temperatura ambiente (Talmacenamiento < $\mathrm{Tg}$ ), sin presentar cambios de fase, debido a que la temperatura de transición vítrea es una propiedad directamente relacionada con la estabilidad durante el almacenamiento (Da Silva Simão et al. 2019). Cabe resaltar, que la estabilidad de los productos alimentarios, bajo un estado cristalino, se debe a la movilidad molecular reducida, lo cual, también está relacionado con la disponibilidad de agua que promueve cambios físicos, químicos y sensoriales en los alimentos (Pereira, 2019).

A partir de los resultados obtenidos, se concluye que la incorporación de la cáscara de mango posibilita el desarrollo de un producto con humedad intermedia tipo enrollable, no requiriendo refrigeración por su alta temperatura de transición vítrea. Asimismo, mediante la formulación seleccionada, es decir, a partir del de mango variedad azúcar y la adición de 1\% de su cáscara deshidratada, es posible obtener un rollo comestible, con características fisicoquímicas y sensoriales adecuadas, además de contener sustancias bioactivas: vitamina C $(48,38 \mathrm{mg} / 100 \mathrm{~g}$, aportando, aproximadamente, el $60 \%$ de la cantidad requerida de consumo diario) y con una capacidad antioxidante, medida con la metodología DPPH de 63,63\%, que pueden favorecer la salud de los consumidores. Lo anterior abre nuevas posibilidades para el desarrollo de productos alimentarios a partir del mango y, a su vez, el aprovechamiento de subproductos, como la cáscara. 


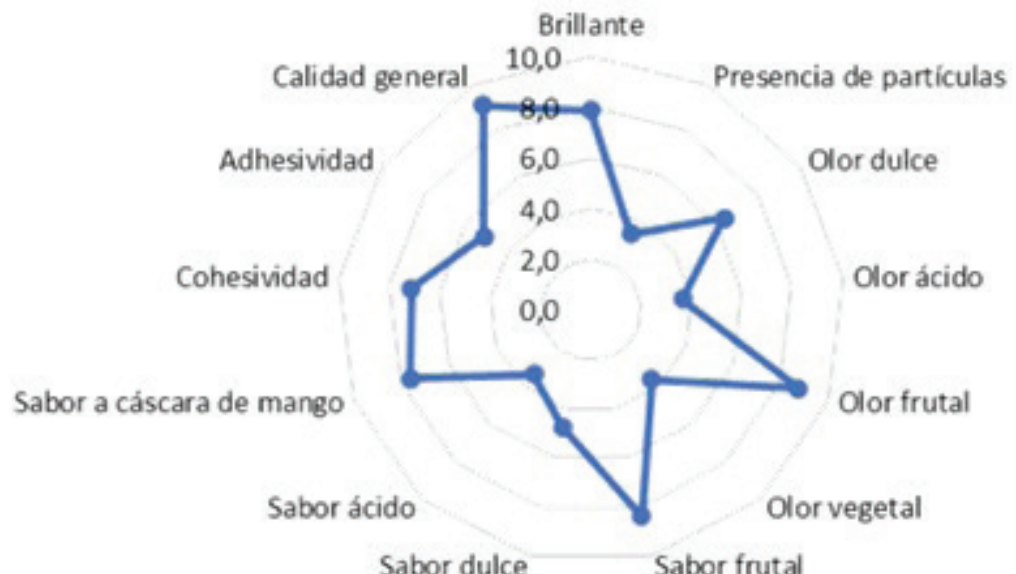

Figura 1. Análisis descriptivo cuantitativo (QDA) del producto desarrollado.

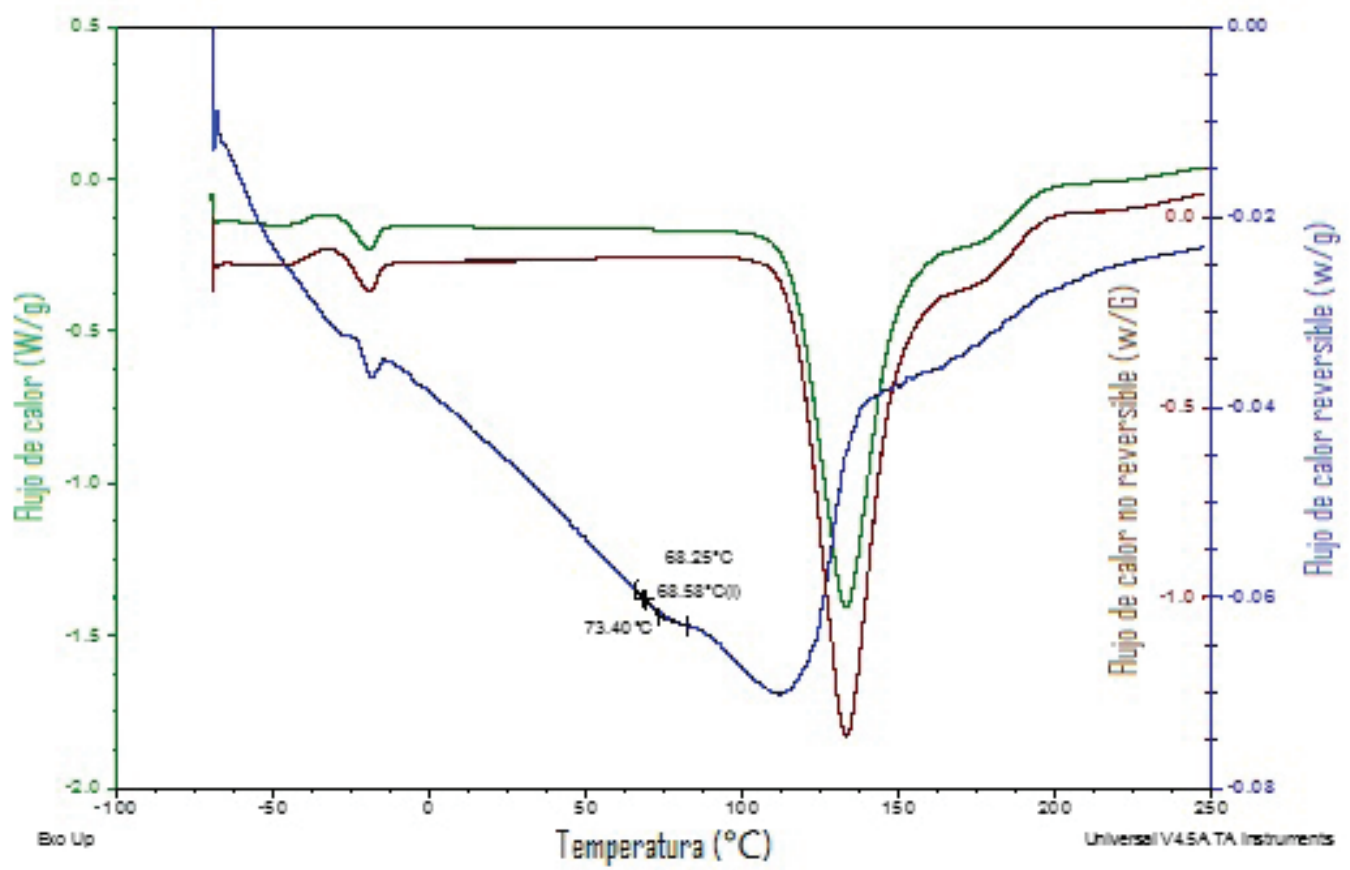

Figura 2. Termograma del producto desarrollado.

Agradecimientos: Los autores expresan sus agradecimientos a Colciencias y la Universidad Nacional de Colombia - sede Medellín, por el financiamiento de la beca-pasantía jóvenes investigadores Convocatoria 2016, así como el apoyo en los recursos requeridos. Conflictos de intereses: El artículo fue preparado y revisado con la participación de todos los autores, quienes declaramos que no existe conflicto de intereses que ponga en riesgo la validez de los resultados presentados. Financiación: Este estudio fue financiado por Colciencias y la Universidad Nacional de Colombia - sede Medellín. 


\section{REFERENCIAS}

1. AJILA, C.; AALAMI, M.; LEELAVATHI, K.; PRASADA, U. 2010. Mango peel powder: a potential source of antioxidant and dietary fiber in macaroni preparations. Innovative Food Science and Emerging Technologies (Holland). 11(1):219224. https://doi.org/10.1016/j.ifset.2009.10.004

2. AKHTAR, H.; RIAZ, A.; SAUD, Y.; ABDIN, M.; CHEN, G.; WAN, P.; ZENG, X. 2018. Production and characterization of CMC-based antioxidant and antimicrobial films enriched with chickpea hull polysaccharides. Internal J. Biological Macromolecules (Holland). 118(1):469-477. https://doi. org/10.1016/j.ijbiomac.2018.06.090

3. CORRALES, A.; MALDONADO, M.; URANGO, L.; FRANCO, M.; ROJANO, B. 2014. Mango de azúcar (Mangifera indica), variedad de Colombia: características antioxidantes, nutricionales y sensoriales. Revista Chilena de Nutrición (Chile). 41(3):312-318. http://dx.doi. org/10.4067/S0717-75182014000300013

4. DA SILVA SIMÃO, R.; OLIVEIRA, J.; GIMENEZ, P.; MATTAR, B.; BORGES, J. 2019. Production of mango leathers by cast-tape drying: Product characteristics and sensory evaluation. LWT - Food Science and Technology (Unites States of America). 99(1):445-452. https://doi. org/10.1016/j.lwt.2018.10.013

5. DAK, M.; VERMA, R.; JAAFFREY, S. 2007. Effect of temperature and concentration on rheological properties of "Kesar" mango juice. J. Food Engineering (Holland). 80(1):1011-1015. https://doi.org/10.1016/j. jfoodeng.2006.08.011

6. DJANTOU, E.; MBOFUNG, C.; SCHER, J.; PHAMBU, N.; MORAEL, J. 2011. Alternation drying and grinding (ADG) technique: a novel approach for producing ripe mango powder. LWT - Food Science and Technology. 44(1):15851590. https://doi.org/10.1016/j.lwt.2011.01.022

7. DORTA, E.; LOBO, M.; GONZALEZ, M. 2011. Reutilization of mango byproducts: study of the effect of extraction solvent and temperature on their antioxidant properties. J. Food Science, 77(1):80-88. https://doi.org/10.1111/ j.1750-3841.2011.02477.x

8. GARCÍA, M.; PAUlA, J.; PAVIANI, L.; CABRAL, F.; MARTINEZ, H. 2015. Extracts from mango peel byproduct obtained by supercritical $\mathrm{CO}_{2}$ and pressurized solvent processes. LWT - Food Science and Technology. 62(1):131-137. https://doi.org/10.1016/j.lwt.2015.01.026

9. GUIAMBA, I.; SVANBERG, U.; AHRNÉ, L. 2015. Effect of infrared blanching on the enzyme activity and retention of $\beta$-carotene and vitamin $C$ in dried mango.
J. Food Science (Holland). 80(6):1235-1242. https://doi. org $/ 10.1111 / 1750-3841.12866$

10. GUIAMBA, I.; AHRNÉ, L.; KHANB, M.; SVANBERG, M. 2016. Retention of -carotene and vitamin $C$ in dried mango osmotically pretreated with osmotic solutions containing calcium or ascorbic acid. Food and bioproducts processing (United Kingdom). 98(1):320-326. https://doi. org/10.1016/j.fbp.2016.02.010

11. FRASCARELI, E.; SILVA; V.; TONON, R.; HUBINGER, M. 2012. Effect of process conditions on the microencapsulation of coffee oil by spray drying. Food and bioproducts processing (United Kingdom). 90(1):413424. https://doi.org/10.1016/j.fbp.2011.12.002

12. INSTITUTO COLOMBIANO DE NORMAS TÉCNICAS, ICONTEC. 1999. NORMA TÉCNICA COLOMBIANA NTC 4592. Productos de frutas y verduras. Determinación del $\mathrm{pH}$.

13. INSTITUTO COLOMBIANO DE NORMAS TÉCNICAS, ICONTEC. 2003. NORMA TÉCNICA COLOMBIANA - NTC 5210. Frutas frescas. Mango. Variedades mejoradas. Especificaciones.

14. JAHURUL, M.; ZAIDUL, I.; GHAFOOR, K.; AL-JUHAMI, F.; NYAM, K.; NORULAINI, N.; SAHENA, F.; OMAR, A. 2015. Mango (Mangifera indica L.) by-products and their valuable components: A review. Food Chemistry (Holland). 183(1):173-180. https://doi.org/10.1016/j. foodchem.2015.03.046

15. KUROZAWA, L.; PARK, K.; HUBINGER, M. 2009. Effect of maltodextrin and gum arabic on water sorption and glass transition temperature of spray dried chicken meat hydrolysate protein. J. Food Engineering (United Kingdom). 91(2): 287-296. https://doi.org/10.1016/j. jfoodeng.2008.09.006

16. LEDEKER, C.; SUWONSICHON, S.; CHAMBER, D.; ADHIKARI, K. 2014. Comparison of sensory attributes in fresh mangoes and heat-treated mango purees prepared from Thai cultivars. LWT - Food Science and Technology. 56(1):138-144. https://doi.org/10.1016/j.lwt.2013.11.011

17. LONDOÑO, J. 2012. Antioxidantes: Importancia biológica y métodos para medir su actividad. En: Garcés, L. (eds). Desarrollo Y Transversalidad. Ed. Corporación Universitaria Lasallista (Medellín). p.128-162.

18. MENDOZA-CORVIS, F.; ARTEAGA-MÁRQUEZ, M.; PÉREZ-SIERRA, O. 2017. Degradación de la vitamina C en un producto de mango (Mangifera indica L.) y lactosuero. Corpoica Ciencia y Tecnología Agropecuria. 18(1):125-137. https://doi.org/10.21930/rcta.vol18_num1_art:563 
19. OLIVEIRA, A.; AMARO, A.; PINTADO, M. 2018. Impact of food matrix components on nutritional and functional properties of fruit-based products. Current Opinion in Food Science (Holanda). 22(1):153-139. https://doi. org/10.1016/j.cofs.2018.04.002

20. ORJUELA, J.; HERRERA, M.; ADARME, W. 2017. Warehousing and transportation logistics of mango in Colombia: A system dynamics model. Revista Facultad de Ingeniería (Colombia). 26(44):73-86. http://dx.doi. org/10.19053/01211129.v26.n44.2017.5773

21. PRABHAKAR, K. 2014. Intermediate Moisture Foods. En: Batt, C.; Tortorello, M. Encyclopedia of Food Microbiology. Ed. Academic Press. Segunda Edición. p.372 - 376.

22. PEREIRA, C. 2019. Phase Transition in Foods. En Gambini, C. Thermodynamics of Phase Equilibria in Food Engineering. Ed. Academic Press. p.421-442.

23. RAHMAN, S. 2009. Food Properties Handbook. Second Edition. CRC Press. (United Stated of America). 860p.

24. ROMERO, D.; CAMBERO, M.; ORDÓÑEZ, J.; DE LA HOZ, L.; HERRERO, A. 2014. Rheological behaviour of commercial cooked meat products evaluated by tensile test and texture profile analysis (TPA). Meat Science (Holland). 98(2):310-315. https://doi.org/10.1016/j. meatsci.2014.05.003
25. SOGI, D.; SIDDIQ, M.; DOLAN, K. 2015. Total phenolics, carotenoids and antioxidant properties of Tommy Atkin mango cubes as affected by drying techniques. LWT Food Science and Technology. 62(1):564-568. https://doi. org/10.1016/j.lwt.2014.04.015

26. SRIWIMON, W.; BOONSUPTHIP, W. 2011. Utilization of partially ripe mangoes for freezing preservation by impregnation of mango juice and sugars. LWT - Food Science and Technology. 44(1):375-383. https://doi. org/10.1016/j.lwt.2010.08.012

27. SUMAYA, M.; SÁNCHEZ, M.; TORRES, G.; GARCÍA, D. 2012. Red de valor del mango y sus desechos con base en las propiedades nutricionales y funcionales. Rev. Mexicana de Agronegocios. 30(1):826-833.

28. 'TEIXEIRA, A.; ARAÚJO, H.; NOGUEIRA, P.; GUITIERREZ, M.; ALMEIDA, A. 2013. Effects of nisin-incorporated films on the microbiological and physicochemical quality of minimally processed mangoes. Internal J. Food Microbiology (Holland). 164(1):135-140. https://doi. org/10.1016/j.ijfoodmicro.2013.04.004

29. WEN, C.; ZHANG, J.; ZHANG, H.; DZAH, C.; ZANDILE, M.; DUAN, Y.; MA, H.; LUO, H. 2018. Advances in ultrasound assisted extraction of bioactive compounds from cash crops - A review. Ultrasonics - Sonochemistry (Holland). 48(1):538-549. https://doi.org/10.1016/j. ultsonch.2018.07.018 